(C) The Author 2015. Published by Oxford University Press on behalf of The British Computer Society. All rights reserved. For Permissions, please email: journals.permissions@oup.com doi:10.1093/iwc/iwv002

\title{
Screening Prototype Features in Terms of Intuitive Use: Design Considerations and Proof of Concept
}

\author{
SANDRINE FiSCHER*, MAKOTO ITOH AND TOSHIYUKI INAGAKI \\ Laboratory for Cognitive Systems Science, Laboratory of Advanced Research B \#0826, Department of Risk \\ Engineering, University of Tsukuba, 1-1-1 Tennoudai, Tsukuba-shi, Ibaraki-ken 305-8573, Japan \\ *Corresponding author: fischer@css.risk.tsukuba.ac.jp
}

\begin{abstract}
Graphical interface use involves schemata operations that range from transfer to induction. The former apply existing knowledge, such as prior schemata, and are effortless, preconscious and intuitive. The latter, which consist in constructing new schemata, are resource-consuming and thus detrimental to intuitive use (IU). A quantitative method is proposed to manipulate and screen schemata operations at the level of an interface's states and features. Relevance for the design cycle of innovative interfaces is critically reviewed, and integration with existing intuitive-use design frameworks is proposed. These considerations are built upon instructional design studies suggesting that assessment should precede and inform the application of design techniques geared toward IU.
\end{abstract}

\section{RESEARCH HIGHLIGHTS}

- Recent design frameworks claim that intuitive use of a device is improved by increasing the familiarity of its features.

- Instructional design studies wam that increased familiarity can be ineffective in cases where people already possess relevant knowledge schemata.

- Incidences of such risk in intuitive-use design are reviewed, along with their prevalence in existing studies of intuitive use.

- An experimental and quantitative method for addressing this issue during the design cycle of innovative interfaces is outlined and illustrated.

- This method screens schemata operations at the level of interface states and features, which allows redesign needs to be pinpointed.

Keywords: Intuitive use; Interaction design theory, concepts, and paradigms; Quantitative methods; User studies; Graphical user interfaces

Received 9 April 2014; Revised 12 December 2014; Accepted 2 January 2015

\section{INTRODUCTION}

A device is 'intuitive' when it may be used as intended, without apparent effort, training or outside help. Recent research has shown that intuitive use (IU) is based on prior familiarity (Blackler et al., 2004; see also Blackler, 2008). Design frameworks based on this finding recommend that device familiarity be increased by thoroughly inspecting users' past experiences and habits (Blackler et al., 2007, 2014; Loeffler et al., 2013). This strategy disregards numerous instructional design studies that found no benefit in increasing familiarity when people already possess relevant knowledge schemata
(Kalyuga, 2007; Mayer, 2001; Sweller et al., 2003). Such studies may explain why several IU and HCI studies have failed to record any usage improvement from increased familiarity. For example, Hurtienne et al. (2013) found that redesigning a ticket vending machine through familiarity principles improved neither its overall usage nor satisfaction. Admittedly, the functionality of a ticket vending machine is well known to most people. Such a case suggests that familiarity-oriented design should focus only on features (e.g. menu, options) incompatible with prior schemata while skipping features that are well known and already compatible with prior schemata. The motivation for 
this strategy is not only to save time in development but also to ensure that redesign efforts translate into tangible benefits.

Modern (e.g. agile) development frameworks recommend that products be specified, developed and tested through short iterations (Ceret et al., 2013). Market characterization (both for purposes of inspiration and differentiation) and user profiling (habits and capabilities) permit early specification of interactive wireframes or prototypes. User tests can then be deployed for identifying 'pain points' and determining which features of the prototype require redesign. Such tests may serve to screen the compatibility of a prototype's features with users' prior schemata. A quantitative operationalization of this rationale, which consists in manipulating users' schemata, is presented in Fischer et al. (2014). The present paper illustrates the bottom line of this approach for user testing. We focus on the function of graphical interfaces, namely the purpose of their features (e.g. print a document, geo-sync a to-do list). In Section 2.1, we outline existing IU design frameworks. The primary goal of our paper is presented Sections 2.2 and 2.3, where we review why design techniques entailed by these frameworks are not likely to be effective for features already compatible with users' prior schemata. This argument is built upon instructional design studies that should be brought to the attention of practitioners desiring to design intuitive devices. In Section 3, we outline a method for assessing users' schemata operations. A concrete application of this method, which is carried out for a multistate interface, is presented in Section 4. Finally, Sections 5 and 6 discuss improvements to the method, and possible integration within the design cycle of innovative interfaces and existing UI design frameworks.

\section{BACKGROUND}

\subsection{IU design frameworks}

To date, two IU design frameworks have been deployed. This section examines their grounds and strategy. To begin, Blackler (2008, see also Blackler et al., 2010) showed that features familiar from other devices tend to be used intuitively. Blackler and Hurtienne (2007) thoroughly reviewed design techniques from $\mathrm{HCI}$ that may promote IU (e.g. stereotypes, metaphors, consistency). In Blackler et al. (2007), these design techniques were integrated within a 'spiral tool' for practitioners to:

Stage 1: select and sample intended user population,

Stage 2: inspect features that are familiar to this population by determining (i) how these features are known, (ii) how they are expected to look, (iii) their location and (iv) their function,

Stage 3: apply these familiarities to designing the features of a device.

Stage 2 (familiarity inspection) aims to extract body reflectors, stereotypes, familiar appearances, functions, locations, affordances and metaphors relevant to the intended user population. This stage combines products reviews, literature reviews, user interviews and usability techniques (e.g. naturalistic observations, participatory design, etc.). Stage 3 (design) is structured so that domain stereotypes (e.g. familiar labels from the domain), metaphors and affordances (e.g. familiar things susceptible to transfer from other domains) and redundancy and consistency can be applied (Blackler et al., 2007). Although not explicitly mentioned, it is expected that user testing occurs after Stage 3. The spiral tool has been deployed for the design and redesign of feature appearance and location for a remote controller, microwave oven and MP3 player (Blackler et al., 2007, 2010, 2014).

Hurtienne (2009) showed that sensorimotor knowledge abstractions, called image schemas, support fast and correct mappings between the layouts and functions of an interface. For example, the image schema UP-DOWN is relevant to designing a volume control or attractiveness meter. Hurtienne's Image Schema CATalog links dozens of these image schemas to hundreds of metaphorical extensions (e.g. UP = 'louder') and user interfaces. One design framework that employs these image schemas is the 'IBIS' method (German for 'design of IU with image schemas', Loeffler et al., 2013), whereby practitioners:

Stage 1: select end-users for systems similar to the one being designed,

Stage 2: conduct surveys and contextual enquiries in which the end-users use the system to be redesigned under thinking-aloud conditions; extract image schemas,

Stage 3: apply these image schemas to specifying a prototype,

Stage 4: evaluate the prototype by means of a questionnaire toolbox.

Stage 2 (contextual inquiry) aims at collecting verbal protocols from which an image schema expert extracts users' image schemata and determines relevant design metaphors. In Loeffler et al. (2013), the IBIS method was deployed to redesign the layout of an image browser and product order manager.

Both the spiral tool and IBIS method place inspection of users' familiarities and habits ahead of any actual design and testing. This strategy has a cost. One of the student designers who tested Blackler et al.'s framework (2007) reported that he spent 'a great deal more time investigating and analyzing the intended users than he would otherwise' (p. 8). One partner who applied Loeffler et al.'s framework (2013) also reported that 'the time required to plan, conduct and transcribe the contextual inquiries was strongly underestimated' (p. 8). In addition, neither framework informs the practitioners as to which features actually require inspection and redesign. For Blackler et al. (2007), designers can enter a spiral at 'a suitable point and leave it when necessary' (p. 8), while for Loeffler et al. (2013) 'image-schematic metaphors that are suitable for

\footnotetext{
'Retrieved March 2014 at http://iscat.stefciu.del.
} 
the implementation are prioritized and afterwards mapped to those use cases where they fit according to the interview' (p. 5). Yet no further guidance is provided for this suitability and prioritization. A more concerning issue, which we now address, are the risks that inspection and implementation of user habits do not translate into substantial improvements.

\subsection{Risks of ineffective design}

Instructional design is the practice of creating experiences that render the acquisition of knowledge and skill more efficient, effective and appealing. Researchers in this field investigate the extent to which instructional techniques support the construction of rich and versatile knowledge. In particular, design techniques are not effective when applied to content that is either too innovative or too familiar. When content is too innovative, people lack the minimum knowledge required to assimilate and elaborate upon it. In such cases, we say that instructional design is inoperative, meaning it fails to support knowledge construction (see Fig. 1). A sensible interpretation of this first risk is that completely novel concepts-even when well formulated-tend to be ignored or falsely interpreted, leading to an incomplete or skewed understanding (Chalmer, 2003).

A second risk is that techniques that are typically beneficial for new content (Borgman, 1999; Hsu, 2006; Mayer, 1976) lose their efficacy when content is too simple or familiar (Hsu, 2005, 2006; Mayer, 1999; Ozgungor and Guthrie, 2004; Pollock et al., 2002). This phenomenon is revealed by factor interactions between prior knowledge (e.g. experts vs. novices) and the design techniques advocated in the IU literature, which include metaphors, stereotypes and redundancy. Metaphors are considered essential to IU, since they represent new concepts in terms of familiar ones (Hurtienne and Blackler, 2007; Loeffler et al., 2013; O'Brien et al., 2010). However, consider a familiar concept $F$, such as a warehouse, used as a metaphor for understanding a technical concept $\mathrm{T}$, such as a database. The metaphor conveyed by $\mathrm{F}$ provides little benefit to a database programmer who already possesses prior knowledge of $\mathrm{T}$. In fact, instructional design research has shown that while metaphors may assist novices in assimilating concepts, they are useless for experts (Hsu, 2006; Mayer, 1999; Mayer, 2001).

Population stereotypes should support IU design since they reflect cultural conventions (Blackler, 2008; Blackler and Hurtienne, 2007). However, when it comes to labeling concepts, Furnas et al. (1987) consider the existence of stereotypes a myth. They found that the likelihood of two people labeling a concept with identical keywords was low (7-18\%). Keyword agreement was also low $(\sim 33 \%)$ for commonly shared topics (e.g. keywords proposed by expert cooks to novice and expert cooks). The same study showed a satisfactory agreement $(50-100 \%)$ only when several words were combined to label a single concept. In fact, expertise (or shared knowledge), which is indispensable to vocabulary stereotypes, entails multiple semantic associations that in turn override stereotypes. This enables experts to easily resolve ambiguities in vocabulary. McNamara and McDaniel (2004) made participants with varying levels of baseball knowledge read sentences containing word ambiguities (e.g. homographs). Those knowledgeable about baseball resolved the ambiguities more quickly than novices. The same phenomenon is corroborated in visual design. Mugge and Schoormans (2012) investigated the effect of stereotypical appearance (e.g. color of a washing machine, shape of a camera) on consumers' a priori usability rating of devices. Their study showed that, compared with products conforming to stereotypes, products whose appearance departed from stereotypes were rated less usable by novices and equally as usable by experts. When combined, the above findings suggest the search for stereotypes may prove more costly than beneficial for products that are common or very familiar.

A similar argument holds for obtaining IU through added redundancy and explanation (Blackler, 2008; Blackler and Hurtienne, 2007). Since novices tend to process information sequentially (Shiffrin and Schneider, 1977), one could argue they would require $n$ times longer to process redundant interfaces, where $n$ is the average redundancy of an interface's features. For example, Reddy et al. (2009) found that a redundant interface slowed down novices with low technological backgrounds. Despite the additional amount of information to process, redundancy should nevertheless increase understanding and comprehension. However, this gain is not expected to occur for contents that are not challenging or innovative to begin with. Research in instructional design shows that novices perform more accurately in the presence of redundancy, yet this gain disappears as they become more experienced (Kalyuga et al., 2000). In particular, Mayer (1999) recommended that redundancy (e.g. using both a word and a picture) be employed for users with low experience rather than high. As for explanations, McNamara et al. showed that adding explanations and coherence to a science text was beneficial only for low-knowledge readers (McNamara, 2001; McNamara and Kintsch, 1996a, b; see also Ozgungor and Guthrie, 2004). In its simplest form, the interaction between prior knowledge and design techniques can be formulated as in Table 1.

In its more complex form, the factor interaction can yield a so-called 'expertise reversal' effect, whereby instructional techniques that are highly effective for novices actually have negative consequences for experts (Sweller et al., 2003; for thorough reviews, see Kalyuga, 2007; Mayer, 2001). For example, Yeung et al. (1998) found that novices learned better from a manual that integrated its vocabulary into the text, as opposed to providing it separately. Experts, though, performed

Table 1. Factor interaction between prior knowledge (expertise) and redesign.

- Novices perform better after design improvement

- Experts perform equally well regardless of design improvement 
less well when the vocabulary was integrated. This phenomenon is particularly detrimental since aside from wasting time attempting to improve something that requires no improvement, redesign actually causes performance deterioration. These two forms of factor interaction are illustrated in Fig. 1.

Both forms of the interaction are conceptualized in terms of cognitive load theory and schemata. Schemata designate the rich, goal-oriented and abstract knowledge representations possessed by experts, yet not by novices (Chi et al., 1981, 1982; Sweller, 2003, 2004). Schemata 'chunk' the processing of information in working memory, which renders processing automatic (Shiffrin and Schneider, 1977; Sweller et al., 2003; van Merriënboer and Sweller, 2005). As a schema is applied 'as is', or 'transferred', its structure overrides the analysis of information and results in minimal effort for comprehension and decision to proceed. This is why experts do not need guidance to intuit and excel at a variety of tasks. Nevertheless, if guidance is added that experts cannot refrain from processing, redesign can result in greater cognitive load and decreased performance (Sweller et al., 2003).

\subsection{Incidence in $I U$ research}

Instructional design studies do not imply that experts knew the tested material, but that their knowledge schemata were sufficiently abstract for subsuming the material. A schema represents the principle(s) common to several experiences at the expense of their specificity. The learning process by which experiences are abstracted into a single schema requires either time or effort. For example, the first time one discovered how to click an icon likely resulted in a specific representation. If exposed to only that one icon, other icons would require effort

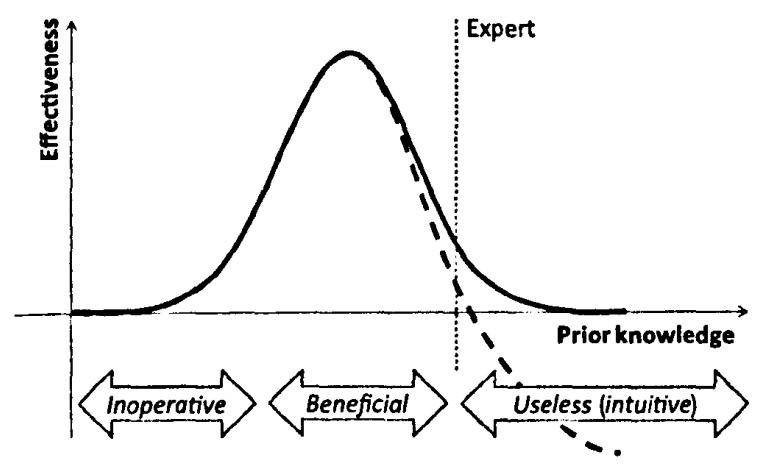

Figure 1. Effectiveness of design techniques (solid) and the expertise reversal effect (dashed), as a function of prior knowledge. The vertical line (dotted) represents the threshold of prior knowledge beyond which one is considered an expert. At lowest levels of prior knowledge, design techniques are inoperative. At intermediary levels of prior knowledge, they become beneficial. At highest levels of prior knowledge (e.g. experts), the content is intuitive and design techniques tend to be either ineffective (solid) or counter-effective (dashed). and deliberation to process. Only by clicking more icons, in different contexts and for other purposes, would one abstract a common schema that subsumes all such experiences past and future. Any icon would then be automatically processed through that same schema. In other words, knowledge schemata are abstract and transferable to a variety of instances (Rumelhart and Ortony, 1977). This argument, which was demonstrated by Gick and Holyoak (1983), Hintzman (1986) and Reber (1967), seems applicable to IU. Blackler et al. (2010) found more IUs from participants with a broad technological background than from those familiar with only a certain type of device. Hurtienne et al. (2013) showed that usability reflects prior experience with various device types rather than one. O'Brien et al. (2012) confirmed through naturalistic analysis that prior knowledge associated with the use of everyday devices has multiple sources. Fischer et al. (2015) found feature functions familiar from other devices and domains to yield the same transfer operation.

We argue along these lines, and the risks outlined in Section 2.2 , that intuitive features are already compatible with prior schemata and should not benefit from redesign. Several findings in IU literature support this argument. Hurtienne et al. (2013) redesigned a ticket-vending machine through metaphors, familiar tabs, clear labels, etc. Contrary to expectation, usage performances and satisfaction did not improve. Perhaps schemata related to purchasing were sufficiently generic to override a variety of instantiations, including the redesign of a vending machine. However, Hurtienne et al. found that participants' prior experience with technology had a greater effect for the original machine than the redesigned one. This outcome resembles the factor interaction in Table 1, which indicates that redesign has a greater effect on participants with low experience rather than high. Such selective effects of redesign, though, are likely to be hindered when participants with different technology experiences are pooled together.

Two other examples of usage accuracy not benefiting from redesign include Gudur et al. (2013), who applied a simpler menu structure to a pet-sitting game, and Blackler $e t$ al. (2014), who applied their spiral tool to the features of a microwave oven. Neither design strategy resulted in improvement of the device's 'correct uses' by participants. The number of 'IU s' coded at the level of participants' think-aloud protocols increased, though. Their coding employed Blackler's five heuristics (2008): verbalized expectations, certainty of correctness, reference to past experience, absence of verbalized reasoning and latency. Interestingly, such a selective effect of design on verbalizations, yet not on accuracy, seems imputable to the schema construct. As discussed by Camp et al. (2001), gradual acquisition of domain schemata (e.g. by education or training) causes a shift in performance criterion from accuracy to speed to automaticity. Novices must rely on short-term memory to interpret, form and test hypotheses about the information they encounter (for classical accounts of automaticity, see Anderson, 1993; Posner and Snyder, 1975). In such cases, accuracy is a primary criterion of performance (van Merriënboer, 1997) and verbalizations tend 
Tuble 2. Factor interaction between prior knowledge (feature familiarity) and redesign.

- Features not compatible with prior schemata are used more correctly after design improvement

- Features compatible with prior schemata are used equally correctly regardless of design improvement

to be detailed (Chi et al., 1981). As domain rules are acquired in long-term memory, performance becomes more accurate. This initially results in improvement of speed (e.g. decision time, completion time), and then development of automaticity (e.g. dual task performance), which ultimately renders verbalizations less detailed and more principle-oriented (Chi et al., 1981, Posner and Snyder, 1975). From this perspective, the design of simple functions (e.g. fecding a pet, heating food, purchasing a ticket) would seem better gauged by speed and verbal protocols rather than accuracy. Conversely, accuracy seems more sensible for assessing the design of innovative functions and cuttingedge technology. Altogether, the fact that redesign modulated the five coding heuristics for Gudur et al. (2013) and Blackler et al. (2014), yet did not increase accuracy, likely stems from the tested devices being fairly common and having basic functionality. Regardless of the criterion, and without a means to diagnose the innovativeness of a device, UI-oriented techniques run the risk of being ineffective.

\section{AN EXPERIMENTAL APPROACII TO SCREENING SCIIEMATA OPERATIONS}

\subsection{Operationalization considerations}

We derived Table 1 from instructional design studies that controlled prior knowledge as an inter-individual factor (i.e. novices and experts are different individuals of an experiment). Interactive products tend to mix common and innovative features, though, so that prior knowledge acts as an intraindividual and intra-material factor. Table 1 can thus be reformulated into Table 2.

In order to operationalize Table 2 , one could redesign an interface for IU and conduct user tests that compare original and redesigned versions as a function of features' prior familiarity. Since the redesign of an entire interface can be overkill, though, one could instead manipulate participants' representation of the original interface through instructions. As for design manipulations, the effectiveness of instructions tailored to the construction of new schemata depends on prior knowledge (Table 3).

As indicated by the dashed cells in Table 3, instructional manipulation allows factor interaction to be addressed with just one version of the material and two experimental groups: one group whose instructions are tailored for construction of new schemata, and one whose instructions are not (control).

\subsection{Schemata operations and patterns}

In instructional design, a variety of manipulations have been devised that support the construction, assimilation or learning of new schemata. Many, such as taking notes vs. listening (Peper and Mayer, 1986), are too educationally oriented for HCI settings. Some manipulations (e.g. Mayer, 1980), however, present traits that are ecological and transposable to $\mathrm{HCI}$. Notably, one could capture screenshots of the states (i.e. distinct feature configurations displayed on the screen) for a graphical user interface and make several groups study these screenshots prior to its use. In this way, Fischer et al. (2014) manipulated amendment of prior schemata and induction of new schemata via three instructional groups:

- a control group, who used the interface without studying its screenshots,

- a reading group, who used the interface after studying every screenshot through 'word matching',

- an induction group, who used the interface after studying every screenshot through 'function matching'.

Word and function matching followed the procedure illustrated in Fig. 2: participants were presented with a clue (word or function), then a screenshot of the interface, and then gave a yes/no judgment based on whether the clue matched the screenshot.

Word clues corresponded to a single word appearing in a screenshot. Thus, word matching required that screenshots be skimmed at a lexical level, which is not likely to support induction of new schemata (for classic evidence that schema induction is not supported by reading, see Gick and Holyoak, 1980; Mayer, 1980). Function clues were sentences that described, in the form of a concrete task, a functional

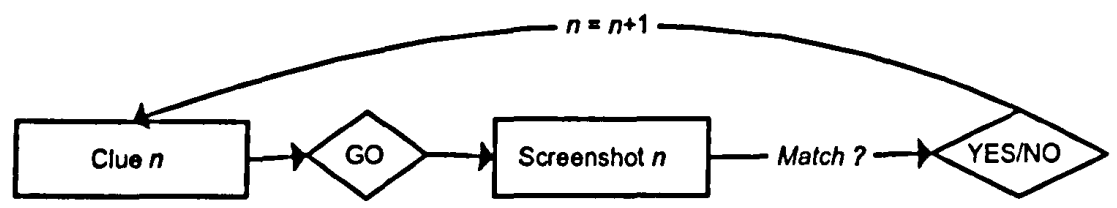

Figure 2. The clue-screenshot matching procedure used for manipulating schema operations. 
Table 3. Examples of design manipulations, instructional manipulations and their pattern of interaction with prior knowledge.

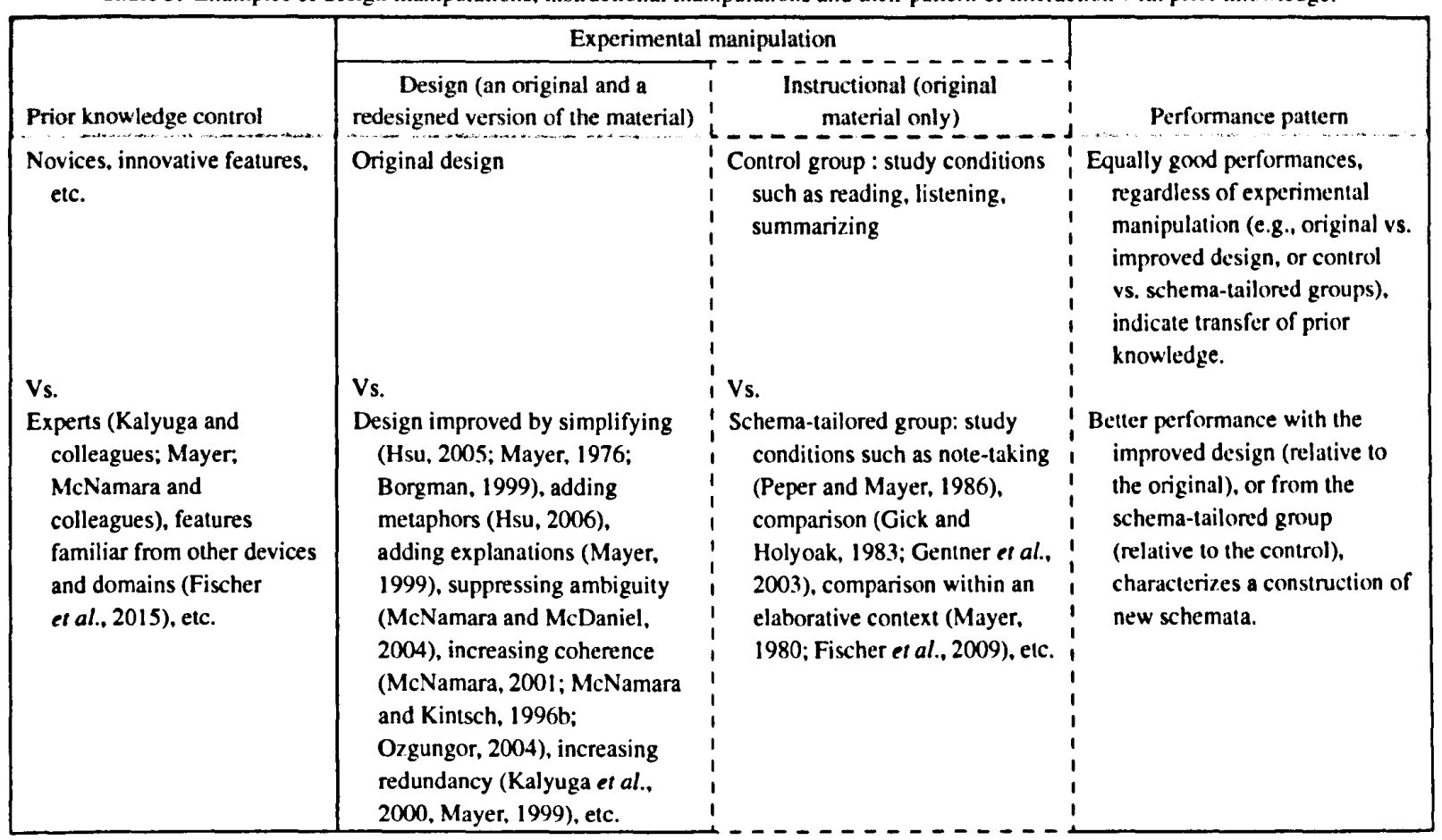

relationship between two features in a screenshot. Function matching incidentally required that screenshot features be interpreted, elaborated and most importantly compared at a functional level. Such comparison has been shown to support the induction of new schemata, namely their construction in a single take (Gentner and Medina, 1998, Gentner et al., 2003, Gick and Holyoak, 1983; Mayer, 1980).

Once every screenshot had been matched, each group (between-subject design) used the interface. Their usage performances (accuracy) were measured, and group differences, or 'patterns', were examined. The control group provided baseline performances (i.e. non-manipulated). Relative to this control, performances of the reading and induction groups were representative of schema amendment and induction, respectively.

Group differences associated with study manipulation tend to be minimal when 'stimulus materials are either very easy or very difficult to learn' (Hyde and Jenkins, 1973, p. 472). Hence, features compatible with prior schemata yield equally good performances from all three groups. This pattern, called prior schemata transfer, occurs when features are functionally familiar from other devices or domains, and characterizes IU (Fischer et al., 2015). One can expect that features incompatible with prior schemata yield errors for the control group. If the induction group fails to induce new schemata through function matching, their performances would be as poor as the control group. This pattern, called inoperative induction, is likely duc to participants being too naive about a domain to benefit from schema-tailored instructions (cf. Fig. 1; Chalmers and Humphreys 2003).

Differences among group performances are more pronounced when schema manipulation is more effective. Features compatible with prior schemata, yet that require some amendments, should affect the reading group relative to the control. Based on classical accounts of schema abstraction (Reber, 1989) and schema theory (Rumelhart and Norman, 1978), we posit that word matching allows for moderately resource-demanding schemata amendment operations, such as abstraction of recurring appearance attributes (e.g. layouts, labels), accretion and tuning of existing schemata. Note, however, that the construct validity and practicality of this pattern has yet to be substantiated. Features whose function does not fall under an existing schema yet still is assimilable should affect the induction group relative to the control. In such cases, the induction group is expected to outperform the control group. This pattern, called positive schema induction, occurs when features have innovative functions whose underlying schemata can to induced through function matching (Fischer $e$ al., 2015).

The aforementioned patterns, and schemata operations they represent, are listed in Table 4 . This table relates our 
Table 4. Correspondences between experimental groups, schema manipulations, patterns and redesign.

\begin{tabular}{|c|c|c|c|c|c|}
\hline & Study phase & $\begin{array}{l}\text { Screenshot } \\
\text { encoding }\end{array}$ & $\begin{array}{l}\text { Schemata } \\
\text { manipulation }\end{array}$ & Patterns & $\begin{array}{c}\text { Diagnosis and redesign } \\
\text { strategy }\end{array}$ \\
\hline Control group & None & None & None & $\begin{array}{l}\text { Prior schemata transfer if } \\
\text { usage performances are as } \\
\text { good as the reading and } \\
\text { induction groups }\end{array}$ & $\begin{array}{l}\text { State or feature is intuitive. } \\
\text { Redesign is not needed, } \\
\text { and may be } \\
\text { counter-effective }\end{array}$ \\
\hline Reading group & Word matching & Word-level & Amendment & $\begin{array}{l}\text { Prior schemata amendment } \\
\text { if performances are better } \\
\text { than the control group }\end{array}$ & $\begin{array}{l}\text { State or feature is not intuitive } \\
\text { and required shallow } \\
\text { learning from participants. } \\
\text { Redesign is desirable }\end{array}$ \\
\hline Induction group & Function matching & Function-level & Induction & $\begin{array}{l}\text { Positive schemata induction } \\
\text { if performances are better } \\
\text { than the control group. } \\
\text { Inoperative schemata } \\
\text { induction if performances } \\
\text { are as poor as the control } \\
\text { group }\end{array}$ & $\begin{array}{l}\text { State or feature is not intuitive } \\
\text { and required deep learning. } \\
\text { Redesign is required } \\
\text { State or feature is not } \\
\text { intuitive and required deep } \\
\text { learning that did not occur. } \\
\text { Redesign is required }\end{array}$ \\
\hline
\end{tabular}

manipulations to the performance and design considerations summarized in Tables 1-3, and highlights their relevance to the screening of an interface for redesign.

The proposed screening comes down to determining the extent to which the performances of the reading and induction groups improved relative to the control group. By calculating the average values and variances of these usage performances, differences can be quantified and compared through their effect sizes (Fischer et al., 2014). The effect size is a statistic used to estimate the magnitude of a difference between two groups (generally a control and manipulated group). In other words, it constitutes a standard measure for the practical significance of a manipulation (Kelley and Preacher, 2012; McGough and Faraone, 2009). The application of effect size-based screening to a schema manipulation experiment is outlined below.

\subsection{Method overview}

This section presents a method applicable to multistate interfaces (or interactive wireframes, prototypes, etc.). By 'multistate', we mean that the interface may have hundreds of features, of which several at a time are displayed on a screen in configurations called states. The method begins with an experiment consisting of a study and usage phase, shown in the upper part of Fig. 3 and further described in Section 4.2. The study phase requires a stimulus presentation module, while the usage phase requires that participants' actions on the interface be logged. These log files are then parsed and analyzed, as shown in the lower part of Fig. 3.

To prepare the study phase, a screenshot is taken for each state of the interface. The practitioner must generate word and function clues that match half the screenshots and mismatch the other half. Mismatching word clues correspond to a word that does not appear in the state, while mismatching function clues correspond to a function that subtly mismatches the screenshot's features. A stimulus presentation module ${ }^{2}$ (labeled 'Module' in Fig. 3) is needed to display the clues, screenshots and buttons labeled 'YES', 'NO' and 'GO'. As illustrated in Fig. 2, the module must display a clue for a predefined time (e.g. $15 \mathrm{~s}$ ), or until participants click 'GO', after which the screenshot to be matched is displayed. Participants give their matching judgments by clicking the 'YES' or 'NO' buttons.

The usage phase is prepared by defining a scenario of usage tasks to be performed on the interface. Each usage task requires participants to navigate from a current state to a goal. This activity consists of recursively determining which feature in the current state best reduces the distance to the goal. Because such determination is prone to error and correction, accuracy is inversely proportional to the number of features selected or states explored during a given usage task. While one may count these actions by hand, such as from a video, a more convenient way is to automatically log them from the interface. To this effect, we employed a toolkit called Automatic Mental Model Evaluator (AMME; Rauterberg, 1993), as well as Visual Basic for Applications (VBA) macros. With an interface $\mathrm{V} / \mathrm{O}$ description file (i.e. a listing of the interface's features, commands and feature-command-feature triplets), AMME automatically formats the command actions stored in a $\log$ file into matrices and action sequences. ${ }^{3}$ These outputs are then parsed by VBA macros that count participants' actions

\footnotetext{
${ }^{2}$ This module may either be hand written code (e.g. Matlab, $\mathrm{C \# )}$ or a software package (e.g. Psyscope, e-Prime) that presents stimuli and records participants' responses.

${ }^{3}$ Details available on July 2014 at http://www.idemployee.id.tue.nl/g.w.m. rauterberg/amme.html.
} 


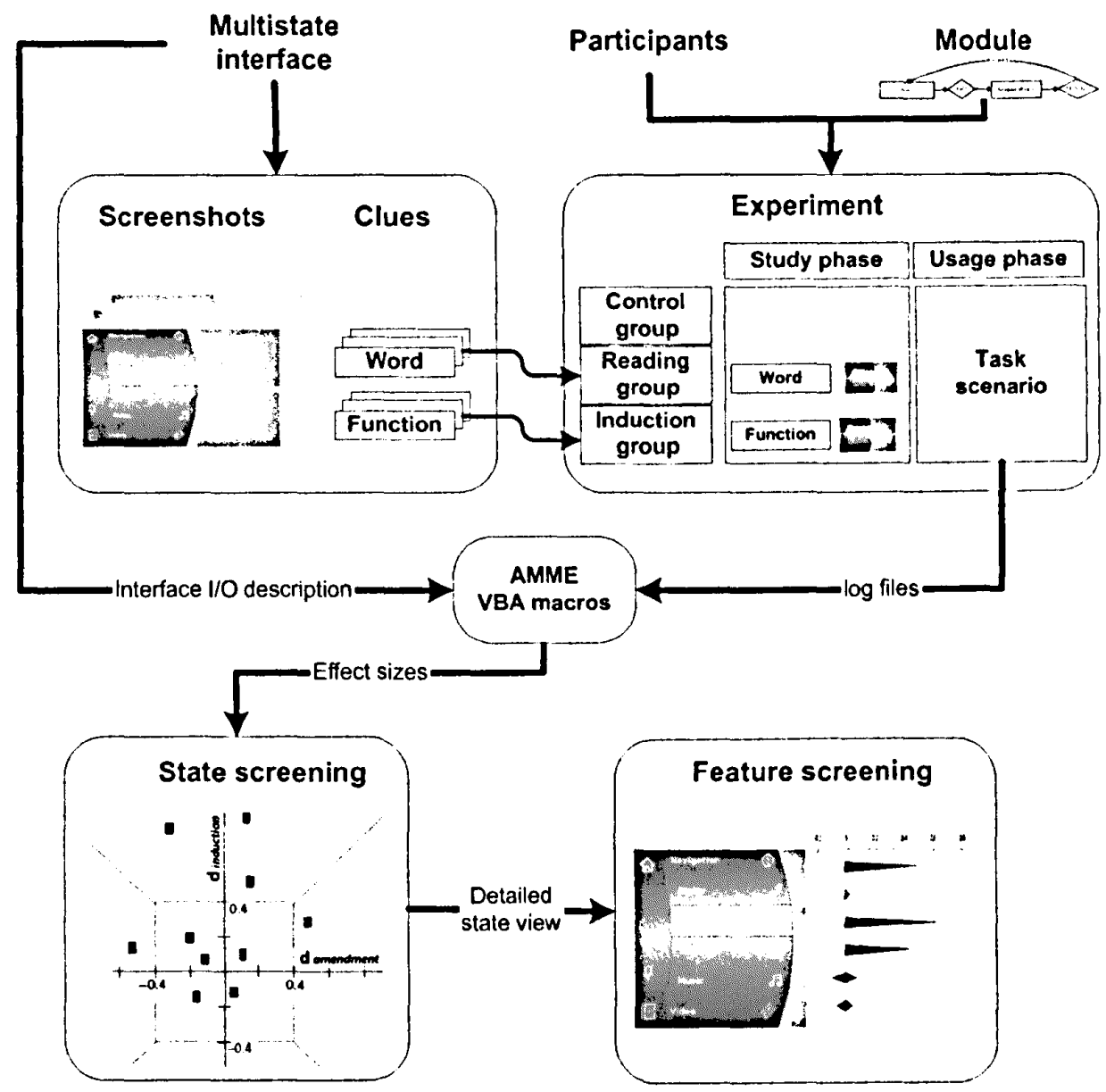

Figure 3. Experimental procedure and screening method, extended from Fischer et al. (2014a). AMME is Rauterberg's Automatic Mental Model Evaluator (1993), VBA is Visual Basic for Applications and V/O is input/output.

by feature, state, or usage task, which then serve to screen performances at any of these granularities.

The screening process begins by calculating mean values and variances of each group's performances. For a given granularity (e.g. data points corresponding either to features, states or usage tasks), effect sizes can be determined in terms of Cohen's $d$, which is a standardized mean difference. ${ }^{4}$ With the control group as a baseline, $d_{\text {amendment }}$ is calculated between the control and reading groups, and $d_{\text {induction }}$ between the control and induction groups. The values for $d_{\text {induction }}$ vs. $d_{\text {amendment }}$ are then plotted. As a rule of thumb for interpreting effect sizes, Cohen (1988) considered a $d$ of 0.2 as small, 0.5 as medium and 0.8 as large. Based on these values, it is reasonable to consider the effect size as moderate-to-large for states having

\footnotetext{
${ }^{4}$ The Cohen's $d$ for two distributions is the difference of their means divided by their pooled standard deviation.
}

$d>0.4$. Values that fall within the range $-0.4<d<0.4$ correspond either to transfer or inoperative induction, meaning the control and induction groups performed equally well or equally poorly, respectively. To distinguish between these two cases, the control group's best and worst performances, or lowest and highest action counts, should be represented with different colors/markers in this region of the 2D scatterplot (see 'State screening' in Fig. 3, and also Fig. 5). Determination of these bes $/$ worst cutoff values depends on data distribution, project needs and available resources'. In the following section, we showcase this screening for a concrete prototype interface. This proof of concept conveys the capacity of our screening

\footnotetext{
${ }^{5}$ For example, for an entertainment interface one may chose to redesign only $15 \%$ of the states yielding the most errors. Altematively, for interfaces used in high-risk environments, one may want to redesign every state above some error threshold.
} 
method to provide interface analytics that support redesign strategies at any desired level of granularity.

\section{PROOF OF CONCEPT}

\subsection{Tested interface}

The method developed above is applied to an automobile onboard computer prototype. Onboard computers aggregate multiple services into a multistate interface whose $\mathrm{V} / \mathrm{O}$ structure is hidden, complex and likely to puzzle novice users. They place enormous emphasis on function attributes by integrating vehicle, infrastructure and satellite technologies with entertainment and telecommunication services. Different brands and models of onboard computers may vary not only in their technological services, but also in the way these services are presented, labeled and controlled. As a result, even with a simple rental car one may encounter functions too innovative to be understoond on the spot.

Our experiment used an onboard computer prototype called DoIT\#. The features of this prototype were determined by revicw of existing onboard computers, along with analysis of R\&D trends (for details about the prototype specification, see Fischer, 2010), and they range from common (e.g. dial a call, defrost the windshicld) to speculative (e.g. wireless retrieval of advertisements). DoIT\# was interfaced with a state window and command panel, and comprised 75 states similar to the one illustrated in the upper part of Fig. 4.

The interaction principle underlying DoIT\# was that of a hicrarchical navigation, commonly found not only in onboard computers, but also in web sites, smart-phone apps, etc. In Fig. 4, the labels 'Vehicle Security Functions', 'Car checking', etc., correspond to distinct features that, when selected (viz. ENTER), change the current state into another state displaying its own features. Features are selected by clicking on the

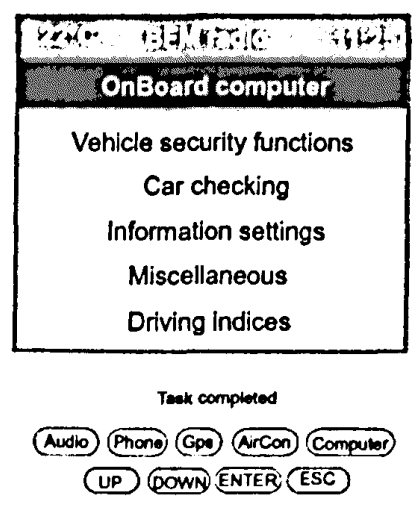

Figure 4. DoIT\#' state window (top) and command panel (bottom). command panel with a mouse. These clicks were recorded in a $\log$ file, counted using AMME and VBA macros, and then averaged to compute effect sizes. Since participants clicked on features, which were embedded in states, and states were explored during tasks, their performances could be analyzed at any of one of these granularity levels.

\subsection{Participants and procedure}

This analysis extends a previous study conducted on 30 Japanese students at the University of Tsukuba, Japan (Fischer $e t$ al., 2014). Prior research has shown that IU is moderated by age and technological familiarity, yet not by gender (Blackler, 2008; Blackler et al., 2010). Thus, while it is essential to recruit participants from the same age bracket (e.g. 18-39) and technological background, gender can be considered irrelevant. In our case, a preliminary survey was used to select participants who possessed a driver's license, did not study Computer Science and had never used an onboard computer or (global positioning system) GPS navigation system. Age of participants ranged from 18 to 27 years, and gender did not play a role in our selection.

The experiment was administered by slideshow, which participants browsed at their own pace. Participants' cognitive style was assessed by means of the Rational Experiential Inventory (Epstein and Pacini, 1999; Naitou et al., 2004; for details see Fischer et al., 2015) and evenly matched across the experimental groups. Participants in the control group proceeded directly to the usage phase. Participants in the reading and induction groups viewed an animated slide describing word or function matching, after which they matched the screenshots of DoIT\#. For all the three groups, the usage phase was introduced by a brief explanatory slide. An experimenter then intervened to demonstrate two basic usage tasks and provide each participant a handout listing 10 usage tasks to perform on their own. The tasks included (i) displaying the number of kilometers traveled, (ii) requesting the route to the house of a friend whose contact information is in the address book, (iii) requesting a route that avoids tolls, (iv) displaying the navigation directions in the rear-view mirror, (v) setting the temperature to $18^{\circ} \mathrm{C}$, (vi) activating a sleeping alert, (vii) activating assistance for passing cars, (viii) activating the internal air filter, (ix) setting the ventilation to silent mode and (x) calculating rest time during the trip. In order to simulate realistic usage conditions, no performance requirements were specified, and no success feedback was provided. Participants who could not finish a task notified the experimenter, who directed them to start the next task.

\subsection{State and feature screening}

Effect sizes can be calculated for any level of granularity (e.g. per state, feature or usage task), any subset of data (e.g. all usage 


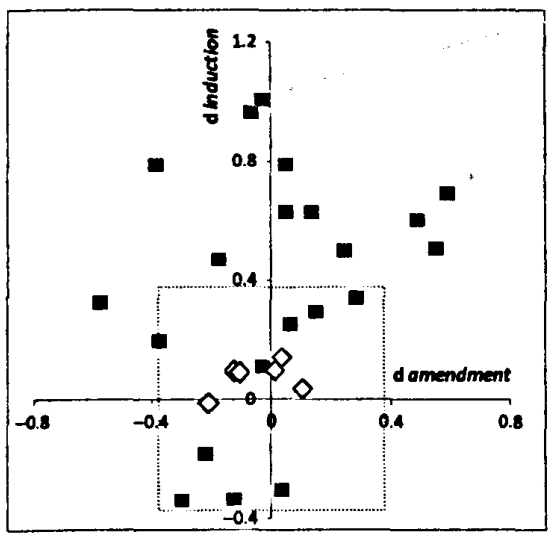

'Guidance' state, of. Fig. 7

'Driving ossistance' stote, cf. Fig. 6

Figure 5. State screening in terms of $d_{\text {induction (greater than }-0.4) \text { and }}$ $d_{\text {amendment }}$ States in the low-effect size region (red dotted box) yield either an inoperative induction pattern (red squares) or transfer pattern (green diamonds)

tasks, only tasks involving innovative features) and any criterion of task success (e.g. only tasks successfully completed). As previously stated, these choices are based on project needs and resources. For example, we chose to screen the states of DoIT\# that were explored by participants during tasks that involved automotive technology, regardless of task success. ${ }^{6}$ We specifically targeted states that yielded a large positive induction pattern. Indeed, while states yielding either large positive or inoperative induction patterns require redesign, the former would seem more straightforward to reformulate since one can draw from the function clues by which participants induced schemata. In Fig. $5, d_{\text {induction }}>-0.4$ is plotted vs. $d_{\text {amendment }}$ for states explored during tasks $1,2,3,6,7$ and 10, which are the six tasks of our usage scenario aimed at automotive technology (e.g. not air conditioning). Each data point in this scatterplot represents a pair of effect sizes corresponding to amendment and induction for one state of DoIT\#. States inside the low-effect size region (red dashed box) have both $\left|d_{\text {induction }}\right|,\left|d_{\text {amendment }}\right|<$ 0.4 and correspond to either inoperative induction or transfer patterns, the latter of which are colored light green. The cutoff for the transfer pattern was set to one or less state exploration by the control group, on average. States with positive induction $\left(d_{\text {induction }}>0.4\right)$ are located above the red dashed box, while those yielding positive amendment patterns $\left(d_{\text {amendment }}>0.4\right)$ are located to its right.

\footnotetext{
${ }^{6}$ One may limit the analysis only to usage tasks that participants successfully completed. Indeed, participants may not have completed certain tasks because they did not understand the assignment. In experimental psychology, where the purpose is to understand the mechanism of cognition, focus is often on tasks completed successfully. In usability settings that intend to evaluate a prototype, however, one would consider any case where the prototype caused difficulty for participants, and therefore analyze both successful and unsuccessful tasks.
}

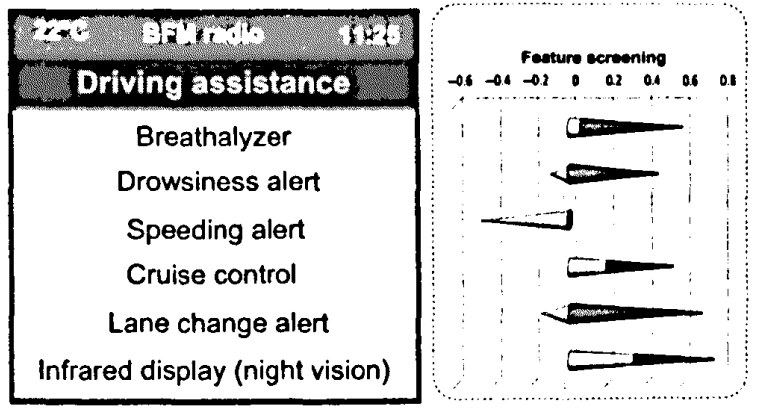

Figure 6. Detailed state view and feature screening for the 'Driving assistance' state (right). Red (dark) is the value of $d_{\text {induction }}$ and blue (light) is the value of $d_{\text {amendment }}$ for each corresponding feature on the left.

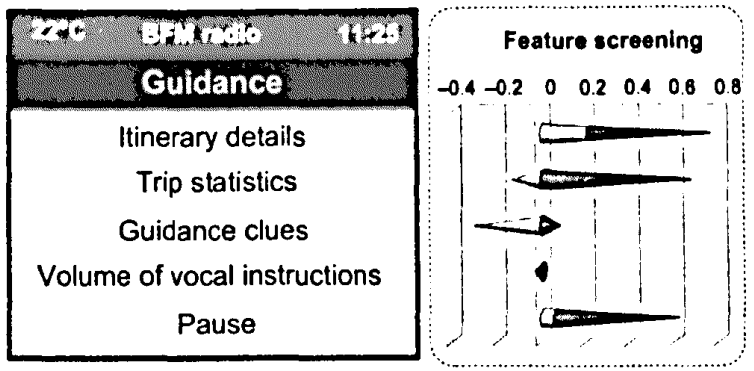

Figure 7. Detailed state view and feature screening for the 'Guidance' state (right). Red (dark) is the value of $d_{\text {induction }}$ and blue (light) is the value of $d_{\text {amendment }}$ for each corresponding feature on the left.

States with large values of $d_{\text {induction }}$ typically require some redesign: they yielded errors for the control group that were overcome only by the induction group, thanks to the schemata induced by the latter. Since each state consisted of several features, it might be useful to screen an interface at the level of its features. By plotting DoIT\#'s features in the same manner as its states, one may prioritize the redesign of features yielding problematic patterns, such as inoperative and positive induction. Alternatively, one could use state scatterplots such as Fig. 5 to instead select the states exhibiting these patterns, and then screen the corresponding features. Screenshots for two of these states, indicated in Fig. 5, are displayed in Figs. 6 and 7 alongside the Cohen's $d$ values for their features. In these figures, light blue corresponds to prior schemata amendment and dark red to new schemata induction.

Figure 6 displays the state titled 'Driving assistance'. The third feature from the top, speeding alert, yielded a negative ${ }^{7}$ amendment pattern, suggesting its function was fairly

\footnotetext{
${ }^{7}$ Patterns of negative induction and/or amendment may reflect an inconsistency between the prototype's information archilecture and users'
} 
compatible with the control group's prior schemata. The first, second and fifth features yielded induction patterns, suggesting their functions challenged participants' prior schemata. These features, in particular lane change alert, required our participants to induce new schemata, and may thus prove challenging for actual users to assimilate.

Figure 7 displays the state titled 'Guidance'. The two first features from the top, Itinerary details and Trip statistics, and the last feature, Pause, yielded positive induction patterns, which suggests that their functions challenged participants' prior schemata. However Pause, being a common-sounding feature, would normally not be expected to yield an induction pattern. By scrutinizing our data at the level of usage tasks, we identified that participants in the control and reading groups mistook the GPS navigation's Pause feature for a calculation of the time at rest, which is a separate feature of the onboard computer. Participants in the induction group did not make this mistake, though, which suggests that function matching helped them to resolve the functional difference between pausing the GPS and displaying the time spent at rest during a trip. This example illustrates how schemata manipulations can complement classical user tests. A test of one control group would only have shown that features with apparent similarities, such as Pause and time-at-rest, can be mistaken. Only by adding a reading and induction group can the severity of this mistake be captured, namely whether the confusion is resolved through amendment of prior schemata or induction of new schemata.

Both the state scatterplot (Fig. 5) and detailed state view (Figs. 6 and 7) correspond to steps for screening an interface in terms of amendment and induction operations. Thorough assessment of an interface may require a successive layering of such screenings in a diagnostic fashion. This rationale is not unlike the way web analytics go beyond simple averages of website visits or traffic. Critical business decisions can be made when traffic is analyzed at finer granularities since it becomes possible to determine the impact of specific marketing campaigns and other events. Of course, averages of each group performance are needed for assessing our experimental manipulation. However, by considering the magnitude of these manipulations (viz. effect sizes) at finer granularities, patterns of schemata operations can be diagnosed for individual states and features of the interface. This can considerably facilitate the formative evaluation of large interfaces, as one is provided with quantitative and cognition-centric guidance regarding the states/features that should be redesigned. As the instructional design studies presented in Section 2.2 suggest, priority should be given to redesign of features with larger induction patterns (e.g. Lane change alert, Drowsiness alert, Trip statistics). Depending on project resources, this redesign may involve current IU design frameworks, as well as numerous techniques

functional schemata. Here, we did not examine this issue: it has yet to be aduressed in a hypothetico-deductive fashion, and it requires triangulations between state, feature and task granularities beyond the present analysis. devised in instructional design (e.g. illustrations, explanatory pop-ups, interactive tutorials).

\section{PERSPECTIVES}

\subsection{Evaluation}

A schema induction experiment can determine the extent to which states or features of an interface challenge the prior schemata of a user population. Such an assessment depends on the sample of participants, which must be carefully selected to meet the demographics and characteristics of expected end users. We selected young drivers who were familiar with common technologies, yet not with GPS systems and onboard computers. One expects that a different population would exhibit a different state scatterplot than the one presented in Fig. 5. For example, users naive with computers and smart phones would be expected to exhibit more inoperative induction patterns, and fewer positive induction and amendment patterns. Or, users familiar with onboard computers would be expected to exhibit more transfer patterns and fewer positive and inoperative induction patterns. The degree to which our screening method captures the prior schemata and learning needs of a user population of interest is an issue of sensitivity to examine in the future.

Methodological improvements are pending, such as the construct validity of each pattern. This work has already begun for patterns of transfer and positive induction, and is expected to extend to the patterns of inoperative induction, negative induction and negative amendment. For now, the latter are based on assumptions from literature that have yet to be addressed in a hypothetico-deductive fashion. Additional studies are needed to extend the scope of our method's application, in particular regarding its sensitivity to individual factors, interface type, etc. Most important is verification of our method in terms of formative assessment, namely its ability to inform redesign and, if so, its cost-effectiveness compared to other lightweight techniques (e.g. cognitive walkthroughs, questionnaires).

\subsection{Design cycle integration}

The present paper discussed the risk of design techniques being ineffective-even counter-effective-for features that fit users' prior schemata. Such risk may be attenuated by prototype testing before heavy deployment of IU design techniques, namely:

Stage 1: designing a prototype,

Stage 2: testing the prototype and determining which novel, innovative or challenging features should undergo Stage 3,

Stage 3: deploying IU design techniques (e.g. prior familiarity, habit inquiry), then iterating the process.

Stage 1 should incorporate well-established practices within the product domain (e.g. mobile vs. desktop), such as stakeholder enquiry, user-requirement and competitor analysis, information 
architecture design, interaction design patterns, usability principles, etc.

Stage 2 requires assessment techniques that are tailored to IU, familiarity or performance. To this effect, practitioners would conduct a schema manipulation experiment and pattern screening, such as the one presented here, or consider other approaches for IU assessment, such as coding of verbal protocols (Blackler, 2008; Blackler et al., 2010; Gudur et al., 2013; Lawry et al., 2010), self-rating questionnaires (Mohs et al., 2006; Naumann and Hurtienne, 2010) or performance metrics (e.g. click behaviors and task paths, time, correct uses, Blackler, 2008; O'Brien et al., 2010). As discussed in Section 2.3, the choice of method carried out at Stage 2 should take into account the relative novelty of the functions being designed. Van Merriënboer (1997) and Camp et al. (2001) have argued that when introducing complex and innovative domain skills to novices, the most important performance criteria is accuracy. In this vein, design of innovative functions, or cutting-edge technology, should first be assessed in terms of accuracy. The reason why initial iterations of prototype testing should employ a schema induction experiment, rather than mere measurements of performance, is because causes of error cannot be objectified by performance alone (Fischer $e t a l$, 2014). To circumvent this issue, researchers in HCI often ask participants to introspect about their behavior (verbal protocol analysis, surveys; for a review of this trend in IU research, see Blackler et al., 2011). These approaches suffer the drawback of being prone to subjectivity from both the experimenter and participants. A screening method that is experimental and strictly quantitative provides more objective insight into user cognition. As a prototype's flaws have been pinpointed and consistently corrected through redesign iterations, other approaches may suffice for monitoring and consolidating enhancements. If improvements are successful, speed and automaticity would become more sensible assessment criteria.

Stage 3 concerns existing IU frameworks, usability recommendations and more. Either the spiral tool or IBIS method, discussed in Section 2.1, would be deployed at this stage. A third and as yet untested framework has been formulated by O'Brien et al. (2010) and approaches IU design in a balanced and encompassing fashion. This framework recommends that designers consider which features of a device are best designed for analysis, and which are best designed for intuition. This viewpoint is one that we adhere to: technology, connectivity and automation inherent to smart phones, homes and cars require a realistic determination of which features can and should be intuitive, and which should be considered from a different angle such as learning or training. O'Brien et al. (2010) related this reflection to Hammond's (1996) analysis-intuition continuum, and proposed to address it heuristically. For instance, practitioners can ask themselves questions (p. 98) such as 'what kind of cues will individuals be examining?', 'Can a task be cleanly decomposed into discrete steps?' and 'Should users be aware of their cognitive activity?'. We propose a means for simultaneously determining which features are intuitive (transfer pattern) and which require an analysis effort, with the latter ranging from supporting (viz. positive/negative induction patterns) to impeding learning (viz. inoperative induction pattern). As such, our method outlines a data-driven and cognition-centric alternative to heuristic approaches to the intuition-analysis continuum.

\section{CONCLUSION}

IU is commonly imputed to prior knowledge factors such as past experience, habits or familiarity. One straightforward application of this idea is that designers may render devices and interfaces more intuitive by making their features closer to what users have experienced in their past, making them more familiar, etc. Yet instructional design studies have raised a flag about this strategy. Notably, effective techniques oriented toward familiarity require neither that people possess relevant abstract schemata nor be too naive about the domain. When these conditions are not met, the design techniques lose their effectiveness and, in certain cases, prove to be counter-effective (viz. expertise-reversal effect).

The cognitive and behavioral outcomes associated with different levels of prior knowledge thus draw a more complex picture than 'the more familiar, the more intuitive'. In light of this issue, it is important to differentiate the features of an interface compatible with users' prior schemata from those that are not. Only the latter, whose usage and assimilation are detrimental to IU, are worth redesigning. On the one hand, this paper illustrates how to pinpoint this compatibility in terms of schemata operations involved during first-time use of prototypes and at design levels that can be acted upon by practitioners (viz. features, states). Such screening may optimize prototype redesign by ensuring that IU design techniques are only deployed for the most deserving features. Future research is still needed, though, to fully establish the application scope of the manipulation and screening of schemata operations.

On the other hand, this paper advocates a diligent incorporation of user tests into current IU frameworks, and IU design in general. Not only are user tests indispensible, they should be conducted prior to application of IU design techniques. Continuing in this vein, practitioners should scale assessment criteria and redesign expectations according to the relative innovativeness of their design projects. Arguably, performance speed and think-aloud protocols that Blackler et al. (2011) praised for researching IU seem sensible for devices that are common and do not pose major first-use difficulty. However, research has found that no improvements in performance (Blackler $e t$ al., 2014; Hurtienne et al., 2013) or even satisfaction (Hurtienne et al., 2013) ought to be expected from redesigning such devices for IU. Conversely, accuracy and schemata operations would better assess interfaces that contain many features 
and/or innovative technology, and are thus prone to first-usage errors. By rendering such features more intuitive, users would experience less failure and disorientation, and thus be in better position to appraise the new technology. Thus far, though, IU design techniques have been tested on devices having basic functionality (remote control, microwave oven, ticket-vending machine, etc.), meaning the goal of rendering innovative technology intuitive remains a challenge in this field.

\section{FUNDING}

The present research was supported by the G-COE Cybernics Program in the University of Tsukuba.

\section{REFERENCES}

Anderson, J.R. (1993) Rules of Mind. Lawrence Erlbaum Associates, New Jersey.

Blackler, A. (2008) Intuitive Interaction with Complex Artefacts: Empirically-Based Research. VDM Verlag. Saarbricken, Germany.

Blackler, A. and Hurtienne, J. (2007) Towards a unified view of intuitive interaction: definitions, models and tools across the world. MMIInterakt., 1, S36-S54.

Blackler, A., Popovic, V. and Mahar, D. (2004) Studies of Intuitive Use Employing Observation and Concurrent Protocol. Design 2004 8th Int. Design Conf., Dubrovnik, Croatia.

Blackler, A., Popovic, V. and Mahar, D. (2007) Developing and Testing a Methodology for Designing for Intuitive Interaction. Int. Association for Societies of Design Research 2007: Emerging Trends in Design Research, Hong Kong.

Blackler, A., Popovic, V. and Mahar, D. (2010) Investigating users' intuitive interaction with complex artefacts. Appl. Ergon., 41, 72-92.

Blackler, A., Popovic, V., Lawry, S., Reddy, R.G., Doug Mahar, Kraal, B. and Chamorro-Koc, M. (2011) Researching Intuitive Interaction. Paper Presented at the IASDR2011, the 4th World Conf. Design Research, Delft.

Blackler, A., Popovic, V. and Mahar, D. (2014) Applying and testing design for intuitive interaction. Int. J. Des. Sci. Technol., 20, 7-26.

Borgman, C.L. (1999) The user's mental model of an information retrieval system: an experiment on an prototype online catalog. Int. $J$ Hum. Comput. Stud., 51, 435-452.

Camp, G., Paas, F., Rikers, R. and van Merrienboer, J. (2001) Dynamic problem selection in air traffic control training: a comparison between performance, mental effort and mental efficiency. Comput. Hum. Behav., 17, 575-595.

Ceret, E., Dupuy-Chesa, S., Calvary, G., Front, A. and Rieu, D. (2013) A taxonomy of design methods process models. Inf. Softw. Technol., 55, 795-821.
Chalmer, P. (2003) The role of cognitive theory in Human-Computer Interaction. Comput. Hum. Behav., 19, 593-607.

Chalmers, P.A. and Humphreys, G.W. (2003) Experimental manipulation of prior experience: effects on item and associative recognition. Memory 11, 233-246.

Chi, M.T.H., Feltovich, P. and Glaser, R. (1981) Categorization and representation of physics problems by experts and novices. Cogn. Sci., 5, 121-152.

Chi, M.T.H., Glaser, R. and Rees, E. (1982) Expertise in Problem Solving. Advances in the Psychology of Human Intelligence. Lawrence Erlbaum Associates, New Jersey, pp. 7-75.

Cohen J. (1988) Statistical Power Analysis for the Behavioral Sciences (2nd edn). Lawrence Erlbaum Associates, New Jersey.

Epstein, S. and Pacini, R. (1999) Some Basic Issues Regarding Dual-Process Theories from the Perspective of CognitiveExperiential Self-Theory. In: Chaiken, S. and Trope, Y. (eds), Dual-Process Theories in Social Psychology. The Guilford Press, New York.

Fischer, S. (2010) Etude diagnostique de l'intuitivité des interfaces innovantes basée sur les principes de transfert et d'induction des schémas. Application aux ordinateurs de bord automobiles. Université Toulouse Le Mirail, Toulouse, France.

Fischer, S., Itoh, M. and Inagaki, T. (2009) A Cognitive Schema Approach to Diagnose Intuitiveness: An Application to Onboard Computers. Proc. 1st Int. Conf. Automotive User Interfaces and Interactive Vehicular Applications, Essen, pp. 35-42.

Fischer, S., Itoh, M. and Inagaki, T. (2014) Identifying the cognitive causes of Human Error through experimentation. Eur. J. Autom., 48/4-6, 319-338.

Fischer, S., Itoh, M. and Inagaki, T. (2015) Prior schemata transfer as an account for assessing the intuitive use of new technology. Appl. Ergon., 46, 8-20.

Furnas, G.W., Landauer, T.K., Gomez, L.M. and Dunais, S.T. (1987) The vocabulary problem in human-system communication: an analysis and a solution. Commun. ACM, 30, 964-971.

Gentner, D. and Medina, J. (1998) Similarity and the development of rules. Cognition, 65, 263-297.

Gentner, D., Loewenstein, J. and Thompson, L. (2003) Learning and transfer: a general role of analogical encoding. J. Educ. Psychol., 95, 393-408.

Gick, M.L. and Holyoak, K.J. (1980) Analogical problem solving. Cogn. Psychol., 12, 306-355.

Gick, M.L. and Holyoak, K.J. (1983) Schema induction and analogical transfer. Cogn. Psychol., 15, 1-38.

Gudur, R.R., Blackler, A., Popovic, V. and Mahar, D. (2013) Ageing, Technology Anxiety and Intuitive Use of Complex Interfaces, 8119 edn. Lecture Notes in Computer Science: Human-Computer Interaction-INTERACT 2013, pp. 564-581.

Hammond, K.R. (1996) Human Judgment and Social Policy. Oxford University Press, Oxford.

Hintzman, D.L. (1986) Schema abstraction in a multiple-trace memory model. Psychol. Rev., 93, 411-428. 
Hsu, Y.C. (2005) The long-term effects of integral versus composite metaphors on experts' and novices' search behaviors. Interact. Comput., 17, 367-394.

Hsu, Y.C. (2006) The effects of metaphors on novice and expert learners' performance and mental-model development. Interact. Comput., 18, 770-792.

Hurtienne, J. (2009) Image Schemas and Design for Intuitive Use: Exploring New Guidance for User Interface Design. Technische Universitat, Berlin, Germany.

Hurtienne, J., Horn, A.-M., Langdon, P. and Clarkson, J. (2013) Facets of prior experience and the effectiveness of inclusive design. Univers. Access Inf. Soc., 12, 297-308.

Hyde, T.S. and Jenkins, J.J. (1973) Recall for words as a function of semantic, graphic, and syntactic orienting tasks. Journal of Verbal Learning and Verbal Behavior 12, 471-480.

Kalyuga, S. (2007) Expertise reversal effect and its implications for learner-tailored instruction. Educ. Psychol. Rev., 19, 509-539.

Kalyuga, S., Chandler, P. and Sweller, J. (2000) Incorporating learner experience into the design of multimedia instruction. J. Educ. Psychol., 92, 126-136.

Kelley, K. and Preacher, K.J. (2012) On effect size. Psychol. Methods, $17,137-152$.

Lawry, S., Popovic, V. and Blackler, A.L. (2010) Identifying Familiarity in Older and Younger Adults. Design Research Society Int. Conf. 2010: Design \& Complexity, Montréal.

Loeffler, D., Hess, A., Maier, A., Hurtienne, J. and Schmitt, H. (2013) DeveJoping Intuitive User Interfaces by Integrating Users' Mental Models into Requirements Engineering. Proc. 27th Int. BCS Human Computer Interaction Conf., London.

Mayer, R.E. (1976) Some Conditions of Meaningful Learning for Computer Programming : Advance Organizers and Subject Control of Frame Order. J. Educ. Psychol., 68, 143-150.

Mayer, R.E. (1980) Elaboration techniques that increase the meaningfulness of technical text: an experimental test of the learning strategy hypothesis. J. Educ. Psychol., 72, 770-784.

Mayer, R.E. (1999) Research-based principles for the design of instructional messages. The case of multimedia explanations. Doc. Des., 1, 7-20.

Mayer, R.E. (2001) Multimedia learning. Cambridge University Press, New York.

McGough, J.J. and Faraone, S.V. (2009) Estimating the size of treatment effects: moving beyond $P$ values. Psychiatry, 6, 21-29.

McNamara, D.S. (2001) Reading both high-coherence and lowcoherence texts: effects of text sequence and prior knowledge. Canad. J. Exp. Psychol., 55, 51-62.

McNamara, D.S. and Kintsch, W. (1996a) Are good texts always better? Interactions of text coherence, background knowledge and levels of understanding in learning from texts. Cognit. Instr., 14, $1-43$.
McNamara, D.S. and Kintsch, W. (1996b) Learning from texts: effects of prior knowledge and text coherence. Discourse Process., 22. 246-288.

McNamara, D.S. and McDaniel, M.A. (2004) Suppressing irrelevant information: knowledge activation or inhibition? J. Exp. Psych. Learn. Mem. Cognit., 30, 465-482.

Mohs, C., Hurtienne, J., Scholz, D. and Rötting, M. (2006) Intuitivität: definierbar, beeinflussbar, uberprüfbar! Düsseldorf, pp. 215-224.

Mugge, R. and Schoormans, J.P.L. (2012) Product design and apparent usability. The influence of novelty in product appearance. Appl. Ergon., 43, 1081-1088.

Naitou, M., Suzuki, K. and Sakamoto, A. (2004) Development of rational and intuitive information-processing style inventory. Japanese J. Pers., 13, 67-78.

Naumann, A. and Hurtienne, J. (2010) Benchmarks for Intuitive Interaction with Mobile Devices. Mobile HCl'10, Lisboa. pp. $401-402$.

O'Brien, M.A., Rogers, W.A. and Fisk, A.D. (2010) Developing an Organizational Model for Intuitive Design. HFA-TR-1001 ed. Georgia Institute of Technology School of Psychology - Human Factors and Aging Laboratory, Atlanta, GA.

O'Brien, M.A., Rogers, W.A. and Fisk, A.D. (2012) Understanding Age and Technology Experience Differences in Use of Prior Knowledge for Everyday Technology Interactions. ACM Trans. Accessible Comput., 4, Article 9.

Ozgungor, S. and Guthrie, J.T. (2004) Interactions among elaborative interrogation, knowledge, and interest in the process of constructing knowledge from text. J. Educ. Psychol., 96, 437-443.

Peper, R.J. and Mayer, R.E. (1986) Generative effects of note-taking during science lectures. J. Educ. Psychol., 78, 34-38.

Pollock, E., Chandler, P. and Sweller, J. (2002) Assimilating complex information. Learning and Instruction 12,61-86.

Posner, M.I. and Snyder, C.R.R. (1975) Attention and Cognitive Control. Information Processing and Cognition: Loyola Symp. Lawrence Erlbaum Associates, New Jersey.

Rauterberg, M. (1993) AMME: an automatic mental model evaluation to analyse user behaviour traced in a finite, discrete state space. Ergonomics, 36, 1369-1380.

Reber, A.S. (1989) Implicit learning and tacit knowledge. J. Exp. Psychol., : General 118, 219-235.

Reber, A.S. (1967) Implicit learning of artifcial grammars. J. Verbal Learn. Verbal Bchav., 6, 863.

Reddy, G.R. Blackler, A., Popovic, V. and Mahar, D. (2009) Redundancy in Interface Design and its Impact on Intuitive Use of a Product in Older Users. Proc. IASDR 2009: Rigor and relevance, Seoul, Korea, p. 209.

Rumelhart, D.E. and Norman, D.A. (1978) Accretion, Tuning and Restructuring: Three Modes of Learning. Semantic Factors in Cognition. Lawrence Erlbaum Associates, Hillsdale, pp. 37-53.

Rumelhart, D.E. and Ortony, A. (1977) The Representation of Knowledge in Memory, pp. 99-135. 
Shiffrin, R.M. and Schneider, W. (1977) Controlled and automatic information processing: II. Perceptual learning, automatic attending and a general theory. Psychol. Rev., 84, 127-190.

Sweller, J. (2003) Evolution of human cognitive architecture. Psychol. Learn. Motiv., 43, 215-266.

Sweller, J. (2004) Instructional design consequences of an analogy between evolution by natural selection and human cognitive architecture. Instnuct. Sci., 32, 9-31.

Sweller, J., Ayres, P.L., Kalyuga, S. and Chandler, P. (2003) The expertise reversal effect. Educ. Psychol., 38, 23-31. van Merriënboer, J.J.G. (1997) Training Complex Cognitive Skills: A Four-Component Instructional Design Model for Technical Training. Engelwood Clifis, New Jersey.

van Merriënboer, J.J.G. and Sweller, J. (2005) Cognitive load theory and complex learning: recent developments and future directions. Educ. Psychol. Rev., 17, 147-177.

Yeung, A., Jin, P. and Sweller, J. (1998) Cognitive Ioad and learner expertise: split-attention and redundancy effects in reading with explanatory notes. Contemp. Educ. Psychol., 23, $1-21$. 